\title{
THE SEMI-SIMPLICIAL FREE LIE RING
}

\author{
BY \\ JAMES W. SCHLESINGER(')
}

1. Recent developments in semi-simplicial topology give rise to a new method for computing the homotopy groups of a simplicial complex $X$. This method, as the results of this paper indicate, should, in time, both extend and unify the existing theory. Briefly we associate with $X$, a spectral sequence $E(X)$ whose $E^{1}$ term depends only on the homology of $X$ and is the homotopy group of a semisimplicial free Lie ring. The $E^{\infty}$ term is the graded group associated with a filtration of $\pi_{*} X$.

This paper is devoted to the computation of a part of the $E^{1}$ term when $X$ is a sphere. This part is essentially a set of multiplicative (under composition) generators for the whole $E^{1}$ term. This fact was discovered in joint work with D. M. Kan and E. B. Curtis and has led to the complete computation of the $E^{1}$ term, which will appear later. As an application of this work we give a new proof of Serre's theorem [8] on the finiteness of the higher homotopy groups of spheres.

This paper is organized as follows:

The second section is an extended introduction which explains in some detail the connection between the homotopy groups of a space and the homotopy groups of certain free Lie rings. The third section develops a few purely algebraic results (in the category of Abelian groups), which, because of their naturality, have topological content (in the category of semi-simplicial Abelian groups). In the last section we attack the problem of computing

$$
E^{1}\left(S^{n+1}\right) \approx \pi_{*} L K(Z, n) \approx \pi_{*} \bigotimes_{r=1}^{\infty} L^{r} K(Z, n) .
$$

When $r$ is an odd prime $p$ we obtain:

$$
\pi_{n+q} L^{p} K(Z, n) \text { is } Z_{p} \text { when } q=2 i(p-1)-1, \quad i=1,2, \cdots,[n / 2] .
$$

2. Background material. A semi-simplicial complex may be regarded as a generalization of a simplicial Łomplex, for the homotopy types of the realizations of the latter form a proper su sset of the homotopy types of the realizations of the former. If $X$ is a connected simplicial complex, then we may choose a reduced semi-simplicial complex $K$ (with just one vertex, the base point) whose realization

Received by the editors May 24, 1965.

(1) This work was partially supported by the United States Army, Durham, North Carolina. 
$|K|$ has the same homotopy type as the realization $|X|$ of $X$. For example if $X$ were an $n$-sphere then $K$ could be the semi-simplicial complex with one vertex *, and one $n$-simplex $x$, together with their degeneracies.

To define the homotopy groups of $K$, we could choose any functor from the category of reduced semi-simplicial complexes to some category on which the homotopy group functor has already been defined, e.g., the realization functor $\mid$ |, [6], to the category of topological spaces with base point gives the most accepted definition. The category of semi-simplicial group complexes is also a category on which homotopy groups have been defined and we may "realize" $K$ in this category by applying the functor $G$ of D. M. Kan. In [5] he defines $G$, a functor from the category of reduced semi-simplicial complexes to the category of (free) semi-simplicial group complexes, and he proves that:

$$
\pi_{n}|K| \approx \pi_{n-1} G K
$$

If we let $A K$ denote the Abelienization of $G K$ then $H_{n}(|K|) \approx \pi_{n-1} A K$ and the homomorphism $\pi_{n} G K \rightarrow \pi_{n} A K$ is the Hurewicz Homomorphism.

In the category of semi-simplicial groups, a short exact sequence, e.g. $[G K, G K] \rightarrow G K \rightarrow A K$, is a fiber space, and induces a long exact sequence of the homotopy groups. Thus the homotopy groups of $[G K, G K]=\Gamma_{2} G K$ and the homotopy groups of $K$ determine the homotopy groups of $K$ up to group extensions. To iterate this procedure we would like to filter $G K$ by subgroups $\Gamma_{n} G K$ so that the successive quotients $\Gamma_{n} G K / \Gamma_{n+1} G K$ are free Abelian, are finitely generated in each dimension when $K$ is finite in each dimension, and depend functorially on $A K$. The lower central series is such a filtration. It is defined by $\Gamma_{1} G K=G K$ and $\Gamma_{n+1} G K=\left[\Gamma_{n} G K, G K\right]$.

Let $L^{r} A K=\Gamma_{r} G K / \Gamma_{r+1} G K$ and $L A K=\bigoplus_{r=1}^{\infty} L^{r} A K$, cf. [2, p. 161] for the proof that $L^{r}$ is a functor from the category of semi-simplicial Abelian groups to the category of semi-simplicial groups. There is a multiplication $[$,$] on$ $L A K$ and it is the free Lie ring of $A K$, cf. [1, p. 285] for an alternate description of $L A K$. The functors $L$ and $L^{r}$ have the property that $\left(L^{r} A K\right)_{q}$ depends only on $A K_{q}$, i.e., there are functors defined on the category of Abelian groups which induce $L$ and $L^{r}$ in the obvious way. Functors with this property are called prolonged functors; they preserve homotopy in the following sense:

If $A$ and $B$ are free Abelian group complexes, $\pi_{*} A \approx \pi_{*} B$ and $T$ is a prolonged functor from the category of semi-simplicial Abelian groups to the category of semi-simplicial Abelian groups then $\pi_{*} T A \approx \pi_{*} T B$, cf. [4, p. 209].

We therefore may choose any free Abelian semi-simplicial resolution of the homology groups of $K$ for our model of $A K$. In particular if $K$ is an $n+1$ sphere, we may choose $A K$ to be the standard model of the Eilenberg-MacLane space $K(Z, n)$, cf. [7, pp. 2-7]. There is, therefore, a homotopy spectral sequence $E\left(S^{n+1}\right)$ relating $\pi_{*}\left(S^{n+1}\right)$ to $\pi_{*} L K(Z, n)=E^{1}\left(S^{n+1}\right)$. 
The fundamental theorem which gives the precise relationship between the spectral sequence $E(X)$ and $\pi_{*} X$ was proved by E. B. Curtis in [3]. It may be simply stated:

The connectivity of $\Gamma_{r} G K$ goes to infinity as $r$ goes to infinity, provided $X$ is simply connected.

This implies that the spectral sequence converges to an $E^{\infty}$ term which is the graded group associated with a filtration of $\pi_{*} X$.

3. Algebraic preliminaries. In this section we shall regard all functors as defined on the category of Abelian groups and taking values in the category of Abelian groups. In so far as the results, which we obtain, are natural, they can be prolonged to the category of semi-simplicial Abelian groups.

Proposition 3.1. If $A$ and $B$ are free Abelian groups then there is a natural isomorphism:

$$
L(A \oplus B) \approx L B \oplus L(A: \otimes T B)
$$

where $T B=\bigoplus_{r=0}^{\infty} B^{r}$ is the tensor ring with unit $\left(B^{0}=Z\right)$.

Proof. By applying the functor $L$ to the maps $B \rightarrow A \oplus B \rightarrow B$, we obtain a direct sum decomposition of $L(A \oplus B)$ into $L B \oplus I$ where $I$ is the kernel of $L(A \oplus B) \rightarrow L B$.

$I$ is a Lie ring and it contains a subgroup $M$ generated by products which contain exactly one factor from $A$ and all other factors from $B$. The group $M$ is isomorphic to $I$ made Abelian, i.e. $M \approx I /[I, I]$. Clearly every homomorphism from $M$ to a Lie ring can be uniquely extended to a ring homomorphism from $I$ to that Lie ring. Hence $I$ is the free Lie ring generated by $M$.

It remains to show that $M$ is isomorphic to $A \otimes T B$. By the anti-commutivity of the multiplication in $I$, we may assume that each product in $M$ is written in the form:

$$
\left[\cdots\left[\left[a, w_{1}\right], w_{2}\right], \cdots, w_{k}\right]
$$

where $a$ is in $A$ and the $w_{i}$ 's are in $L B \subset T B$ (the set of $w_{i}$ 's may be empty). We map this element to:

$$
a \otimes w_{1} \otimes \cdots \otimes w_{k} \text { in } A \otimes T B
$$

(if there are no $w_{i}$ 's we map $a$ to $a \otimes 1$ in $A \otimes T B$ ). This map is well defined since the Jacobi identity holds in both $M$ and $L B \subset T B$. The map is clearly natural and it has an inverse defined by: $a \otimes b_{1} \otimes \cdots \otimes b_{n}$ goes into $\left[\cdots[a, b],, \cdots, b_{n}\right]$.

Definition. If $R$ is.a Lie ring then we define the subrings $\Gamma_{r} R$ by $\Gamma_{1} R=R$ and $\Gamma_{r+1} R=\left[\Gamma_{r} R, R\right]$. These subrings are called the lower central series of $R$. (The direct sum of the successive quotients is again a Lie ring and could be called the associated weighted Lie ring of $R$.) 
Definition. Let $L$ be the free ring functor. We define the functor $J=\Gamma_{2} L / \Gamma_{2} \Gamma_{2} L$ that is $J$ is $\Gamma_{2} L$ made Abelian. The functors $J^{r}$ are defined to be $L^{r} / L^{r} \cap \Gamma_{2} \Gamma_{2} L$ for $r=2,3, \cdots$. It is clear that $J=\bigoplus_{r=2}^{\infty} J^{r}$. (Note also $J^{2} \approx L^{2}, J^{3} \approx L^{3}$.) An alternate definition of $J^{r}$ is given in [2, p. 163].

Proposition 3.2. If $M$ is a free $Z$ module, $r>1$, then there is a natural short exact sequence:

$$
J^{r} M \underset{i}{\rightarrow} M \otimes S P^{r-1} M \underset{j}{\rightarrow} S P^{r} M .
$$

Proof. $S P M$ is the symmetric tensor ring and $S P^{r} M$ is the $r$-fold symmetric tensor product. The map $j$ is the multiplication map of $S P M$ and $i$ is defined on basis elements $\left[\cdots\left[m_{1}, m_{2}\right], \cdots, m_{r}\right]$ of $J^{r} M$ by:

$$
i\left(\left[\cdots\left[m_{1}, m_{2}\right], \cdots, m_{r}\right]\right)=m_{1} \otimes\left(m_{2}, \cdots, m_{r}\right)-m_{2} \otimes\left(m_{1}, m_{3}, \cdots, m_{r}\right) .
$$

It is obvious that $i$ is well defined and one to one. It is also clear that $j$ is onto, $j$ is natural, and $j \circ i$ is zero. To show that $i$ is natural and that the image of $i$ is the kernel of $j$, one may consider $X^{r} M$ the kernel of the natural projection of $T^{r} M \rightarrow S P^{r} M$, where $T^{r} M$ is the $r$-fold tensor product of $M$ with itself. The inclusion $L^{r} M \rightarrow X^{r} M$ induces a natural isomorphism

$$
J^{r} M \approx X^{r} M / M \otimes X^{r-1} M
$$

which in turn is naturally isomorphic to the kernel of $j$. It is easy to show that the composite isomorphism is just the map $i$.

Proposition 3.3. The composite of the natural inclusion $i: L^{r} M \rightarrow T^{r} M$ and the projection $q: T^{r} M \rightarrow L^{r} M$ is multiplication by $r$ when $M$ is free and where $q\left(m_{1} \otimes \cdots \otimes m_{r}\right)=\left[\cdots\left[m_{1}, m_{2}\right], \cdots, m_{r}\right]$.

This may be proved by direct computation.

4. The basic computation. Let $K(Z, n)$ denote the Eilenberg-MacLane complex whose one nonzero homotopy group is $Z$ and occurs in dimension $n$. Since $K(Z, n)$ is a free module in each dimension we may apply various functors (from Abelian groups to Abelian groups) to each dimension of $K(Z, n)$ and obtain new semi-simplicial Abelian groups whose homotopy groups depend only on the homotopy groups of $K(Z, n)$.

We can use Proposition 3.2 to relate the homotopy groups of $J^{r} K(Z, n)$ with those of $S P^{r} K(Z, n)$. A great deal is known about the homotopy groups of $S P^{r} K(Z, n)$ (or $Z_{p} \otimes S P^{r} K(Z, n)$ ). For example in $[4$, p. 306] we have the results:

Proposition 4.1.

$$
\pi_{n+q} S P^{2} K(Z, n) \approx\left\{\begin{array}{l}
Z \text { if } n \text { is even and } q=n, \\
Z_{2} \text { if } q=2,4,6, \cdots, 2[(n-1) / 2] \\
0 \text { otherwise. }
\end{array}\right.
$$


Proposition 4.2. If $p$ is a prime and $0<r<p$ then

$$
\pi_{g}\left(Z_{p} \otimes S P^{r} K(Z, n)\right) \approx\left\{\begin{array}{l}
Z_{p} \text { if } n \text { is even and } q=r n, \\
0 \text { otherwise. }
\end{array}\right.
$$

By the techniques of [4] one can further obtain:

Proposition 4.3. If $p$ is a prime then $\pi_{n+q}\left(Z_{p} \otimes S P^{p} K(Z, n)\right)$ is $Z_{p}$ if $q \equiv 0,1 \bmod 2(p-1)$ and $1<q \leqq n(p-1)$; it is zero otherwise.

In addition to these results, $[4$, p. 307$]$ contains the computation of the homomorphisms:

$$
\pi_{r n} K(Z, n) \otimes S P^{r-1} K(Z, n) \rightarrow \pi_{r n} S P^{r} K(Z, n),
$$

which, when $n$ is even, are just multiplication by $r(Z \rightarrow Z)$. Thus one can apply Proposition 3.2 to prove the following three propositions:

Proposition 4.4.

$$
\pi_{n+q} L^{2} K(Z, n)=\pi_{n+q} J^{2} K(Z, n)=\left\{\begin{array}{l}
Z \text { if } n \text { is odd and } q=n, \\
Z_{2} \text { if } q=1,3, \cdots, 2[n / 2]-1, \\
0 \text { otherwise. }
\end{array}\right.
$$

Proposition 4.5. If $p$ is a prime and $2<r<p$ then

$$
\pi_{q}\left(Z_{p} \otimes J^{r} K(Z, n)\right)=0 .
$$

Proposition 4.6. If $p$ is a prime then $\pi_{n+q}\left(Z_{p} \otimes J^{p} K(Z, n)\right)$ is $Z_{p}$ if $q \equiv-1,0 \bmod 2(p-1)$ and $0<q<n(p-1)$; it is zero otherwise.

Proposition 4.7. If $p$ is a prime then:

$$
\pi_{*}\left(Z_{p} \otimes L^{p} K(Z, n)\right) \approx \pi_{*}\left(Z_{p} \otimes J^{p} K(Z, n)\right) .
$$

Proof. Since the result clearly holds for $p=2$ or 3 , we assume $p \geqq 5$. Consider the lower central series of $Z_{p} \otimes \Gamma_{2} L K(Z, n)$. The associated weighted Lie ring is:

$$
Z_{p} \otimes L J K(Z, n)=L\left(Z_{p} \otimes J K(Z, n)\right)=L\left(Z_{p} \otimes \bigoplus_{r=2}^{\infty} J^{r} K(Z, n)\right) .
$$

Since $Z_{p} \otimes \bigoplus_{r=2}^{\infty} J^{r} K(Z, n)$ and $Z_{p} \otimes\left(J^{2} K(Z, n) \oplus \bigoplus_{r=p}^{\infty} J^{r} K(Z, n)\right)$ are free $Z_{p}$ modules, whose homotopy groups are identical, and since $L$ may be viewed as a prolonged functor from $Z_{p}$ modules to $Z_{p}$ modules, we can conclude that:

$$
\pi_{*}\left(Z_{p} \otimes L J K(Z, n)\right) \approx \pi_{*} L\left\{Z_{p} \otimes\left(J^{2} K(Z, n) \oplus \bigoplus_{r=p}^{\infty} J^{r} K(Z, n)\right)\right\} .
$$

Thus by Proposition 3.1, the associated weighted Lie ring of $Z_{p} \otimes \Gamma_{2} L K(Z, n)$ has for its homotopy groups: 


$$
\pi_{*}\left(Z_{p} \otimes L J^{2} K(Z, n)\right) \oplus \pi_{*}\left(Z_{p} \otimes L\left\{\left(\bigoplus_{r=p}^{\infty} J^{r} K(Z, n)\right) \otimes T J^{2} K(Z, n)\right\}\right) .
$$

However $\Gamma_{2} L K(Z, n)=\bigoplus_{s=2}^{\infty} L^{s} K(Z, n)$ and the ideals $\Gamma_{r} \Gamma_{2} L K(Z, n)$ are homogeneous ideals so that the successive quotients are also weighted (or graded) by $s$. Therefore the homotopy groups of $L^{s} K(Z, n)$, which is finitely filtered by its intersections with these ideals, can be computed from the homotopy groups of its successive quotients, and these successive quotients are direct summands of the associated weighted Lie ring. In particular $\pi_{*}\left(Z_{p} \otimes L^{p} K(Z, n)\right)$ can be computed from a spectral sequence which collapses to just $\pi_{*}\left(Z_{p} \otimes J^{p} K(Z, n)\right)$.

Proposition 4.8. $\pi_{*} L^{r} K(Z, n)$ is a $Z_{r}$ module for $r>2$.

Proof. By Proposition 3.3, the map, multiplication by $r$, of $L^{r} K(Z, n)$ into $L^{r} K(Z, n)$, factors through the $r$-fold tensor power $T^{r} K(Z, n)$ of $K(Z, n)$. Furthermore the nontrivial generator of $T^{r} K(Z, n) \cong K(Z, r n)$, goes into zero under the projection $T^{r} K(Z, n) \rightarrow L^{r} K(Z, n)$ if either $n$ is even or $r>2$. Thus every element of $\pi_{*} L^{r} K(Z, n)$ is of order $r$.

Since $\pi_{*} L^{p} K(Z, n)$ is a $Z_{p}$ module, for $p$ an odd prime, we can apply the universal coefficient theorem to conclude:

Proposition 4.9. If $p$ is an odd prime then:

$$
\pi_{n+q} L^{p} K(Z, n) \approx\left\{\begin{array}{l}
Z_{p} \text { if } q=2 i(p-1)-1, i=1,2, \cdots,[n / 2] \\
0 \text { otherwise. }
\end{array}\right.
$$

Proposition 4.10.

$$
\pi_{n+q} S^{r}=\left\{\begin{array}{l}
Z \text { if } q=0, \\
Z+\text { a finite group if } n \text { is even and } q=n-1, \\
\text { a finite group otherwise. }
\end{array}\right.
$$

Proof. By the convergence of the spectral sequence and the fact that $\pi_{*} L^{r} K(Z, n-1)$ is finite unless either $r=1$ or both $r=2$ and $n-1$ is odd.

\section{REFERENCES}

1.H. Cartan and S. Eilenberg, Homological algebra, Princeton Univ. Press, Princeton, N. J., 1956.

2. E. Curtis, Lower central series of semi-simplicial complexes, Topology 2 (1963), 159-171.

3. - Some relations between homotopy and homology, Ann. of Math. (to appear).

4. A. Dold and D. Puppe, Homologie nicht-additiver Functoren, Anwendungen, Ann. InstFourier (Grenoble) 11 (1961), 201-312. 288-312.

5. D. M. Kan, A combinatorial definition of homotopy groups, Ann. of Math. 67 (1958), 
6. J. W. Milnor, The geometric realization of a semi-simplicial complex, Ann. of Math. 65 (1957), 357-362.

7. J. C. Moore, Algebraic homotopy, Mimeographed notes, Princeton University, Princeton N. J., 1957.

8. J.-P. Serre, Homologie singulière des espaces fibres. III. Applications homotopiques, C. R. Acad. Sci. Paris 232 (1951), 142-144.

TUFTS UNIVERSTTY,

MEDFORD, MASSACHUSETTS 